Stephen M. Matyas

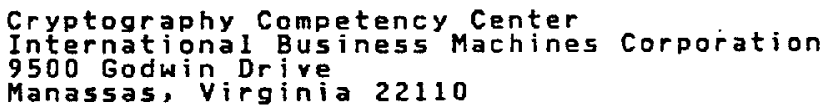

ABSTRACT

A procedure is described for securely initializing cryptographic variables in a large number of network terminals. Each terminal has a cryptographic facility which performs all necessary cryptographic functions. A key distribution center is established, and a public and secret key pair is generated for the key distribution center. Each terminal in the network is provided with a terminal identification known to the key distribution center. The terminal identification and the public key of the key distribution center are stored in the cryptographic facility of each terminal. A terminal initializer is designated for each terminal, and the terminal initializer is notified of two expiration times for the purpose of registering the terminal's cryptovariable with the key distribution center. The cryptovariable is generated by the terminal using its cryptographic facility. Prior to the first expiration time, a registration request is prepared and transmitted to the key distribution center. The registration request includes the terminal identification and the cryptovariable. When the key distribution center receives this request, the cryptovariable is temporarily registered and that fact is acknowledged to the requesting terminal. After the expiration of the second time, the registration is complete. Provisions are also made for invalidating a terminal identification if more than one registration is attempted for a given terminal identification or an intended registration was not made in time.

\title{
BACKGRDUND
}

Historically, contributions in the area of public key distribution can be briefly traced, although a thorough treatment of the subject would naturally include the works of many others. The first known proposal for public key distribution involved placing the public key of each user in a public directory (i.e.. key distribution center) along with the user's name and address [1]. Anyone wishing to communicate with a particular user would first contact the public 
directory and request a copy of that user's public key. A second proposal simply called for a pair of devices wishing to communicate to exchange their public keys via the communication channel in advance of the communication session [2].

An improvement to the first method was made by distributing public keys from a key distribution center (KDC) wherein the messages containing the keying information were "signed" using the secret key of a public/secret key pair belonging to the KDC [3]. In advance, the public key of the KDC is distributed to each communicant in the system and this key is then used to validate the received signature and message containing the keying information. A similar approach was suggested wherein each communicant registers his public key and identifier with the KDC and, in turn, receives from the KDC a public key certificate, which is a message containing his public key and identifier that has been signed using the secret key of the KDC [4]. In advance, the public key of the KDC is distributed to each communicant in the system, and this key is then used to validate received certificates. To communicate, individuals need only exchange and validate each other's public key certificate. Both approaches provide a path with integrity to distribute public keys previously registered with the KDC.

Racal-Milgo proposed a method of dynamic public key distribution which incorporates an anti-spoofing procedure [5]. Briefly, two parties who wish to communicate with a public key algorithm each generate a public and secret key pair. The respective public keys are exchanged via the communication channel. Upan receipt, each communicant calculates a prescribed function of the received public key. The communicants then contact each other via telephone and exchange the calculated values, which can then be verified by the originating communicants. For the check to work, the telephone channel itself must have integrity or the callers must recognize each other's voice.

A similar anti-spoofing technique which pre-dates the Racal-Milgo technique was proposed by Bell Telephone Laboratories 16 J. With Bell's technique, the key validation information is exchanged via letter using the postal system rather than by using voice communications. Otherwise, the concept is the same. For the check to work, the postal system handing the mail must have integrity, otherwise the anti-spoofing check itself could be spoofed. 
From the foregoing, it is apparent that while advances in public key distribution techniques have been made, the problem of initially securely registering public keys with a key distribution center has not been appropriately dealt with.

\section{PUBLIC KEY REGISTRATION PROCEDURE}

It is the object of the public key registration procedure to initialize with security and integrity a large number of devices in an information handing system with cryptographic variables without requiring couriers to transport these cryotographic variables. For convenience, the devices in the network will be terminals. The procedure is general in that it permits the registration of both secret and nonsecret variables, although of primary interest is the registration of public keys of terminals. The public key registration process may also be thought of as part of the larger process of terminal initialization.

Initialization of the terminal is performed by a designated representative called the terminal initializer. In all cases, the terminal initializer is a person who acts responsibly to carry out the steps of the terminal initialization procedure. The terminal initialization procedure comprises the steps of causing the terminal to generate and register one or more cryptovariables with a designated key distribution center (KDC) and promptly reporting to the KDC any encountered problems. Typically, the terminal initializer will be an employee of the organization at the location where the terminal is physically installed, such as a terminal user, terminal owner, manager, or member of the local site security. In situations where a third party key distribution center is emplayed, the terminal initializer may be a locally appointed agent of the KDC. The terminal initializer has no responsibility for transporting keys, public or private, or for installing secret keys by entering them directly into a cryptographic device. Therefore, the terminal initializer is not a courier, and does not perform the functions of a courier.

Each terminal in the network is provided with a cryptographic facility (CF) consisting of hardware and software components that perform the necessary cryptographic functions to support the required cryptographic operations. A subset of these functions support the terminal initialization procedure. Overall cryptographic security, 
including that of the terminal initialization procedure, rests on an assumption of integrity of the CF, including stored keys and programs and associated supporting software, which is guaranteed by the design and by other physical security measures instituted. Prior to the terminal initialization procedure, the KDC generates a public key and secret key pair (PKkdc, SKkdc), which are the keys that operate with the public key algorithm. A unique nonsecret terminal identifier (TID) and the public key of the key distribution center (PKkdc) are assumed to have been installed in the CF of the terminal. The TID and Pkkdc could be installed, for example, in microcode as part of the manufacturing process of the terminal. Alternatively, they could be installed at a central location and the terminals with the installed TID and PKkdc shipped to the final destination, or they could be installed by the terminal initializer, i.e., locally after the terminal has been installed.

For each terminal which is to be initialized, as previously mentioned, the KDC designates a terminal initializer who is responsible for carrying out the necessary terminal initialization procedure at the device. Each terminal initializer is provided with a set of instructions outlining the terminal initialization procedure. The security of the procedure rests on the assumption that the terminal initializer will comply with the issued instructions and understands that failure to comply with these instructions may result in an adversary successfully registering a key with the key distribution center. The KDC also provides to the terminal initializer with two expiration dates, ordinarily separated by several days, which delimit periods of time in which certain prescribed steps within the terminal initialization procedure must be completed. The security of the procedure rests on the assumption that the terminal initializer receives notification of the two expiration dates and the terminal initialization instructions at some time well in advance of the expiration dates so that the steps of the procedure can be performed within the prescribed allotted time.

According to the terminal initialization procedure, prior to the first expiration date, a cryptovariable can be temporarily registered at the KDC under the designated TID provided that the TID has not been invalidated and no other prior cryptovariable has been temporarily registered for the TID. In the discussion that follows, the cryptovariable registered is a public key, and therefore this process 
is called "public key registration." If a public key has already been registered under a given TID, an attempt to register a different public key under that same TID prior to the first expiration date will result in the TID being invalidated. After the first expiration date, the public key registration process is disabled at the KDC for that TID.

Prior to the second expiration date, the KDC permits a TID to be invalidated without "proofn of the identity of the requestor. This process is called "ID invalidation without proof of identity." After the second expiration date, the process of ID invalidation without proof of identity is disabled for that TID, and the temporary status of the registration is considered changed to that of a permanent registration.

After the second expiration date, the KDC permits a TID to be invalidated only after the requestor has been identified and authenticated and his or her authorization to invalidate a particular TID has been verified. This process is called mID invalidation with proof of identity."

After the second expiration date and upon request, the KDC will issue a PK certificate for any TID provided that the TID is valid and a public key has been registered for that TID. A PK certificate consists of a TID, public key, certificate expiration date, possibly other data, and digital signature produced on the foregoing data using the secret key of the KDC. One recommended methad for calculating a signature is to first calculate an intermediate value or function of the message using a strong one-way cryptographic function. This intermediate value is then decrypted with the secret key skkdc to produce the signature. If the TID is invalid or no public key has been registered, an appropriate response message is prepared on which a digital signature is calculated using the secret key of the KDC and the message and signature are returned to the requesting terminal.

Under normal operating conditions, the terminal initialization procedure proceeds as follows. Well in advance of the first expiration date, a public key and secret key pair are generated at the terminal using an available key generation procedure. A public key registration request message containing the TID and public key of the terminal is sent to the KDC. Under normal conditions no adversary 
will have interfered with the process, and therefore no public key will yet be temporarily registered under the designated TID. Therefore, the KDC temporarily registers the public key under the specified TID, prepares an appropriate response message containing the TID and public key on which a digital signature is calculated using the secret key of the KDC in the manner previously described, and the message and signature are returned to the requesting terminal. After authenticating the received message, the requesting device signals the terminal initializer that the desired public key has been temporarily registered at the KDC under the specified TID. (It is assumed that the hardware and software components involved with terminal initialization have integrity and that the terminal being initialized is the prescribed, genuine terminal.) The procedure for authenticating a signature is similar to the procedure for calculating a signature. The same intermediate one way function of the message, which was used in calculating the signature, is again calculated from the message. The receired signature is then encrypted using the public key of the KDC (PKkdC) to recover a clear value of the one way function of the message, and the recovered one way function of the message is compared for equality with the calculated one way function of the message. If the comparison is favorable, the message and signature are accepted; otherwise, if the comparison is unfarorable, the message and signature are rejected.

The protocol now requires a delay, and the terminal initializer must wait for the passage of the second expiration time in order that the KDC may assure that the temporarily registered public key is genuine; i.e., that it originated from the authorized, appointed terminal initializer. After the second expiration time, a terminal-initializer-initiated message containing the $T I D$ is sent to the KDC requesting "ID Verification" for that TID. Under normal conditions no adversary will have interfered with the process and therefore the specified TID will be valid and the previously temporarily registered public key will still be registered. But due to the expiration of the second time, the registration is now considered permanent. Therefore, the KDC prepares and returns a message to the requesting terminal specifying the registered public key for that TID. A digital signature is prepared on this message using skkdc which allows the requesting terminal to authenticate the received message using the installed Pkkde in the manner previously described. This signals satisfactory completion of the terminal 
initialization procedure and provides the necessary proof that the desired public key has been successfully initialized at the kDC. Alternatively, the KDC could return a public key certificate to the requesting terminal, and this would also serve as proof to the terminal that the public key had been registered.

Once an authenticated response has been received from the KDC stating that a public key has been temporarily registered or that the TID has been invalidated, the worst that could happen is that an adversary could cause a genuine temporarily registered public key to be erased by invalidating the TID prior to the second expiration time. Hence, for practical purposes, a safe state is reached, and it is therefore possible with no 1055 in security to allow a protocol variation wherein the terminal-initializer-initiated message sent to the KDC requesting "ID Verification" following the second expiration time can be replaced by a similar terminal-user-initiated message. This protocol variation has the advantage that ordinarily the terminal initializer can complete the terminal initialization procedure with only one terminal visit, orior to T1. The terminal user, who is notified by the terminal initializer of the terminal initialization status and the value of T2, completes the protocol after the second expiration time. Of course, the protocol variation is the same as the original protocol when the terminal initializer and the terminal user are the same person.

In a network where it is convenient for the KDC to send messages to the terminals, such as in a store-and-forward electronic mail distribution system, yet another variation on the protocol is possible. The step following the second expiration time wherein a terminal-initializer-initiated or terminal-user-initiated message is sent to the KDC requesting "ID Verification" is replaced by a step wherein the KDC automatically prepares and sends a response to the original requesting terminal. This response is just the same as that which would have been sent in the response to a request for "ID Verificationn except here the response is triggered by reaching the second expiration time rather than upon receiving a request message. otherwise, the protocol is the same. If no response is received at the terminal within a reasonable period of time after the second expiration time, the terminal initializer or the terminal user, depending on which protocol is used, reports this discrepancy to the KDC. 
An expanded discussion of the above initialization procedure can be found in IBM TR $21.1000\lceil 71$.

\section{REFERENCES}

1. Diffie, W. and Hellman, M. E., "New Directions in Cryptography,"

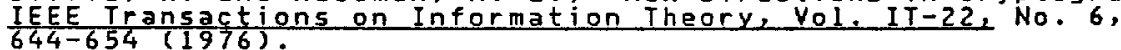

2. Rivest, R:L., Shamir, A, and Adleman, L,, MA Method for Obtaining Digital Signatures and Public-Key Cryptosystems," Communication of the ACM, Vol. 21, No. 2, 120-126 (1978).

3. Needham, R.M. and Schroeder, M.D., "Using encryption for authentication in large networks of computers," communication of the ACM. Vol. 21, 993-999 (December, 1978).

4. Kohnfelder, L. M., "Using Certificates for Key Distribution in a Public-key Cryptosystem, "Massachusetts Institute of Technology, unpublished paper (May 19,1978 ).

5. Abbruscato, C.R., "Public Key Security, "Telecommunications, Vol. 18 , No. 12, 60-64, 72 (December, 1984).

6. Myers, F.H., "Data Link Encryption System," NTC Conference Record, National Telecommunications Conference, Washington, D.C.. New York, NY, 1979, Vol.3, Pp. 43.5.1-43.5.8

7. Matyas, 5.M., Initialization of Cryotographic Variables in a Network with a Large Number of Terminals, IBM Technical Report, TR 21.1000, Kingston, N.Y., August, 1986. 